\title{
Reflexiones en torno a la conservación integrada, compartida y participativa del paisaje cultural. El caso de Paranapiacaba
}

Vanessa Gayego Bello Figueiredo | Facultad de Arquitectura y urbanismo, Universidad de PUC-Campinas, Ex-Subprefeita de Paranapiacaba

URL de la contribución <www.iaph.es/revistaph/index.php/revistaph/article/view/3620>

\section{RESUMEN}

Una relación armoniosa entre la conservación patrimonial y el desarrollo socio-económico es un reto siempre presente en la gestión de sitios históricos. Enfoques recientes, como el de paisaje cultural, se centran en la interdisciplinariedad y en el territorio como claves para la formulación de políticas integradas que superen las dicotomías clásicas, incluyendo el distanciamiento en la gestión de los distintos patrimonios: natural, cultural, material e inmaterial. Tomando como referencia el Programa de Desarrollo Sostenible de Paranapiacaba (Santo André-SP, Brasil), el artículo muestra cómo las políticas sectoriales se diseñaron e implementaron, destacando las enseñanzas extraídas de esta experiencia.

A través de la descentralización administrativa se materializó, entre 2001 y 2008, la integración de las políticas culturales, de conservación medioambiental, turismo comunitario, desarrollo social, planificación urbana y participación ciudadana. Esta experiencia, debido a sus innovaciones y logros, ha sido reconocida por organismos nacionales de Brasil, como el IPHAN y el Ministério das Cidades, y por organismos internacionales como el Programa Mundial de Estudios del Patrimonio de la Universidad Tecnológica de Brandeburgo (Programa World Heritage Studies da Brandenburg University of Technology) y el Laboratorio Internacional de Paisajes Culturales de la UPC-Universidad Politécnica de Cataluña.

\section{Palabras clave}

Conservación (Patrimonio) | Desarrollo socioeconómico | Gestión cultural | Paisaje cultural | Paranapiacaba (Brasil) | Patrimonio ferroviario | Planificación territorial | Políticas públicas | 


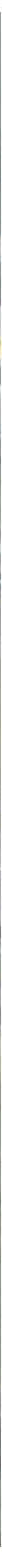

Vila Nova (en primer plano), el patio ferroviario (al lado), y Vila Velha (al fondo). 2006 | fuente LUME FAU USP/PMSA 


\section{LA VILLA FERROVIARIA DE PARANAPIACABA}

Situado en lo alto de la Serra do Mar, a una altitud de 796 metros, a 64 km de la capital del estado, el pueblo ferroviario de Paranapiacaba es un ejemplo destacado del patrimonio cultural y natural de Brasil. Se encuentra dentro de la zona protegida de los manantiales de San Andrés, región donde se conservan fragmentos significativos de Mata Atlántica (bosque tropical), reconocidos en 1994 por la UNESCO como Reserva de la Biosfera del Cinturón Verde de São Paulo.

Paranapiacaba, "lugar desde donde se ve el mar" en lengua indígena, conserva un importante patrimonio tecnológico ligado al ferrocarril y es testimonio de un modelo arquitectónico y urbano bastante avanzado para su momento de aplicación. Este pueblo ferroviario se desarrolló a partir de 1860 con la introducción del tren Santos-Jundiaí, construido por la compañía británica São Paulo Railway. En 1957 el ferrocarril y todos sus activos fueron incorporados a la administración de la Red de Ferrocarril Federal, S. A. A partir de los años 80 del siglo pasado, atravesó por un intenso período de abandono y decadencia, a raíz de la indiferencia de los gobiernos por el transporte y patrimonio ferroviario.

En 1987, se reconoció este patrimonio por parte de la agencia estatal, y en 2002 a nivel nacional (IPHAN ) y también municipal. Entre 2003 y 2007 fue considerado por el World Monuments Fund (WMF) uno de los cien patrimonios en peligro más importantes del mundo. En 2008, en virtud de las políticas de rehabilitación aplicadas para sacarlo de la lista WMF, se convirtió en el primer patrimonio cultural de São Paulo y también el primer patrimonio industrial brasileño en formar parte de la lista presentada por Brasil al título de Patrimonio Mundial de la UNESCO. Esta candidatura fue

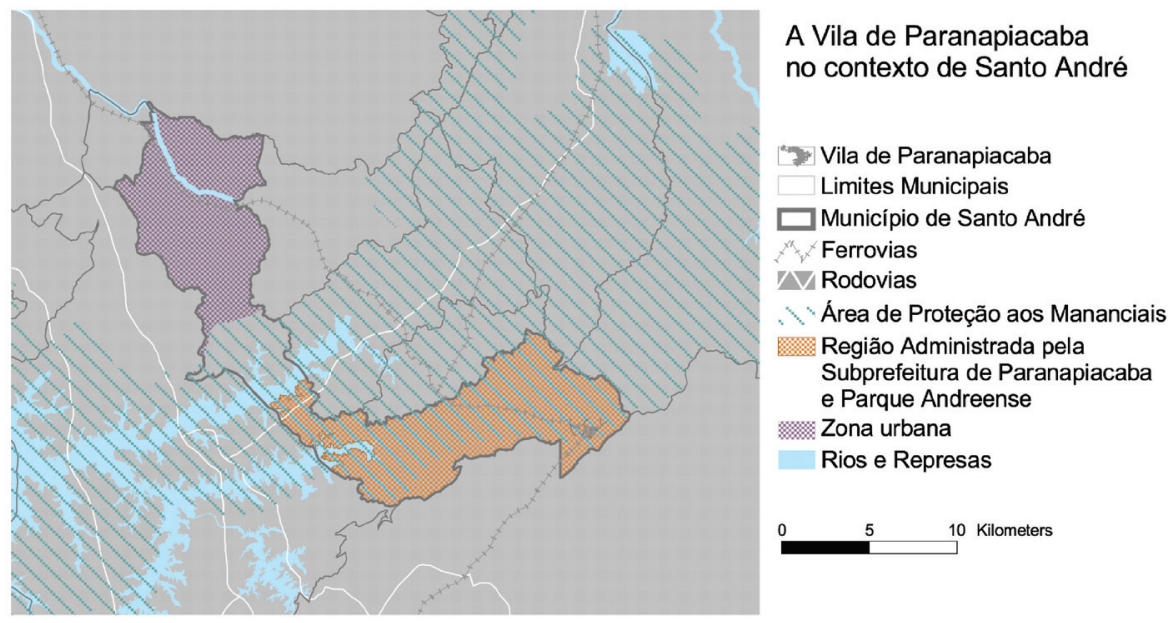

Mapa de Santo André, destacando la zona de los manantiales | fuente LUME FAU USP/PMSA, 2006 
retomada por el IPHAN en 2014, tras el anuncio de la sustanciosa cifra de 15 millones de dólares en inversiones del Programa Nacional de Ciudades Históricas.

La villa ferrroviaria de Paranapiacaba consta de tres zonas con morfología urbana muy distinta: la parte alta, con un patrón de ocupación característico del paisaje colonial portugués, con 247 habitantes (PMSA/SUB, 2008)1; Rabique, una región con fuertes pendientes, alto riesgo de deslizamientos de tierra y de ocupación irregular; y la parte baja con 1.171 residentes. Esta última se divide en tres zonas: el casco antiguo (Vila Velha), donde se ubicaba el campamento de los ferroviarios, con viviendas de adobe, barro y paja, almacenes y talleres, distribuidos desordenadamente a lo largo de la Rua Direita; el patio de ferrocarril, donde hay restos del sistema de dos funiculares que utilizaban máquinas fijas a vapor tiradas por cables de acero en contrapeso. En 1974 se instaló el sistema de piñón de agarre en los raíles del primer funicular, todavía en funcionamiento para el transporte de cargas. Y Vila Nova, o Martin Smith, que había adoptado estructuras urbanas habituales en Europa después de la Revolución Industrial, con un trazado ortogonal, con calles anchas y jerarquizadas, callejones auxiliares y redes de infraestructura urbana. La ciudad obrera con sus diferentes tipos de madera responde a la tradición constructiva inglesa, cuya homogeneidad formal contrasta con la férrea jerarquía social de la empresa. El tamaño y el tipo de las viviendas y sus diferentes acabados definían las distintas categorías de empleados: ingenieros, bomberos, conductores y los alojamientos para empleados solteros.

\section{INTEGRANDO POLÍTICAS: DESCENTRALIZACIÓN, INTERDISCIPLINA- RIEDAD, INTERINSTITUCIONALIDAD, PARTICIPACIÓN Y TERRITORIO}

El aislamiento del distrito de Paranapiacaba, causado por el brazo de la presa Billings que cruza la ciudad, es el primer problema para su integración territorial, convirtiéndose en un desafío la promoción de la descentralización administrativa y de los sentimientos de "pertenencia" e identidad de los 6.399 habitantes (PMSA/SUB, 2008) ${ }^{2}$, aproximadamente el 10\% de la población andreense.

El proceso se inició en 1989 a través de las centrais de atendimento (call centers) (PMSA/SUB, 2006). En 1998 se realizó un proyecto de "Gestión Participativa en Zonas de Cuencas Hidrográficas", en colaboración con la Universidad de la Columbia Británica y la Agencia para el Desarrollo Internacional (Canadá), centrándose en el desarrollo socio-económico, ordenamiento territorial y alternativas para las áreas medioambientales sensibles (PMSA, 2004). En 2001 se creó la subprefectura para favorecer la integración de la zona en la ciudad; compaginar la producción de agua; la preserva-

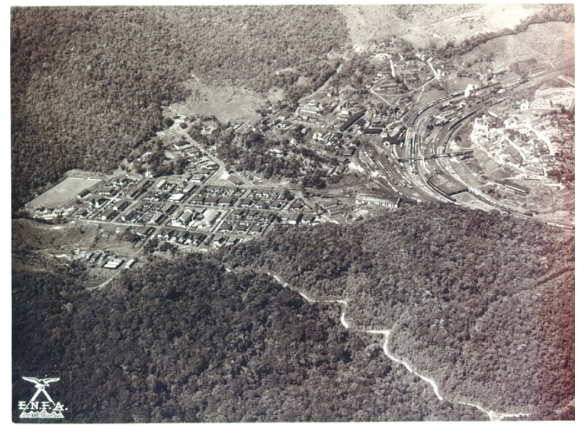

Foto Aérea de Paranapiacaba de 1940 | fuente ENFA-Empresa Nacional de Fotos Aéreas. Gobierno del Estado de São Paulo. Instituto Geográfico e Cartográfico
1

Basado en datos de 2000 del IBGE-Instituto Brasileiro de Geografia e Estatística. 


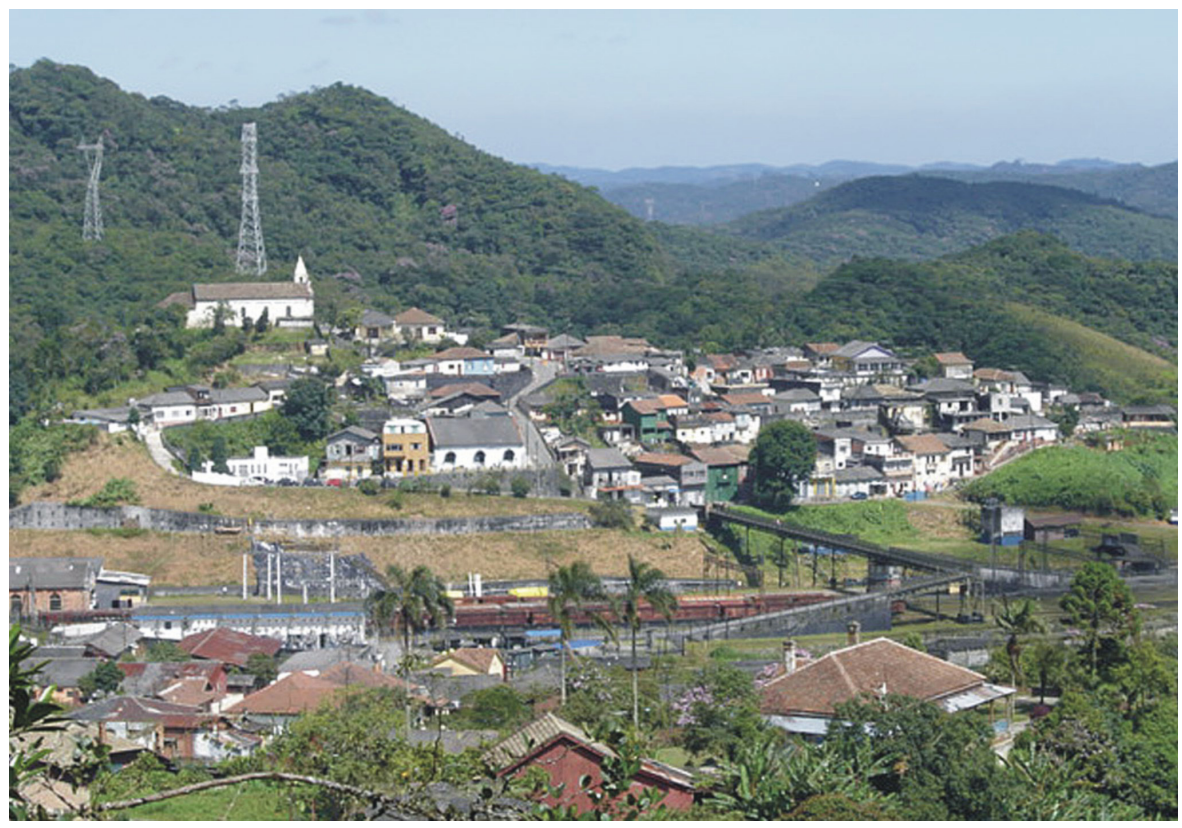

Parte alta de Paranapiacaba, 2005 | foto Vanessa Figueiredo

ción ambiental y cultural; la comunicación; y el turismo comunitario como la principal actividad económica.

Con la compra de la Villa por la administración municipal en 2002, se inició la gestión administrativo-financiera de los inmuebles de propiedad pública y el Programa de Gestión del Desarrollo Local Sostenible, para intensificar el proceso de recuperación de este patrimonio, entendido y tratado como "paisaje cultural".

Este enfoque, adoptado por el Comité del Patrimonio Mundial desde 1992 y por la Recomendación R(95)9 (CONSEJO DE EUROPA, 1995), ha sido recientemente incorporado por el IPHAN (Carta de Bagé, 2007 y Chancela, 2009). Se amplía significativamente la noción de patrimonio, articulando conceptos y objetos de diferentes campos, teniendo en cuenta la interdisciplinariedad y el territorio como claves para superar la fragmentación que todavía se practica tanto en la concepción como en la implementación de políticas públicas.

Partiendo de una concepción integradora de la relación hombre-naturaleza y de los patrimonios material e inmaterial, entender el paisaje como patrimonio admite la relación intrínseca entre las aproximaciones hechas desde la historia, la antropología, la ecología, las artes, etc., y sus correspondencias en el entorno físico, urbano, rural o natural. Esta concepción hace que sea muy compleja la gestión de los patrimonios, y que se requiera la revisión de las políticas de conservación vigentes, sobre todo en su articulación intersecto- 
rial e interinstitucional (FIGUEIREDO, 2014), como confirmará la experiencia de Paranapiacaba.

A continuación presentamos estas políticas integrales, sin detallarlas a fondo, con la intención de construir una visión general de esta experiencia, destacando los principales legados, lecciones, permanencias y rupturas.

\section{LA PLANIFICACIÓN URBANÍSTICA Y LA PRESERVACIÓN PATRIMONIAL}

La práctica de la conservación de sitios históricos urbanos en Brasil ha demostrado que la tutela, sin dejar de cumplir su papel en la puesta en valor, es un instrumento insuficiente ante las necesidades para la preservación de los paisajes culturales. Tal aproximación considera esencial la acción integrada en la planificación y gestión territorial de las políticas ambientales, socioeconómicas y culturales. Por lo tanto, el reto está en combinar la política de conservación con un proceso dinámico de desarrollo de las ciudades, lo que necesariamente implica el no impedir el cambio, sino dirigirlo hacia la perspectiva del desarrollo sostenible.

Teniendo en cuenta estas consideraciones se estableció la Ley de ZEIPP (Zona de Especial Interés del Patrimonio de Paranapiacaba), un instrumento que ha venido siendo considerado como innovador por el Ministério das Cidades y el IPHAN ${ }^{3}$, especialmente por articular el planeamiento urbano según las directrices de conservación y gestión del paisaje cultural de Paranapiacaba y demás políticas sectoriales.

La ZEIPP fue creada en 2004 por el Plan Director Participativo de San Andrés (Ley 8696) y regulada en el 2007 (Ley 9018). Se trata de una simbiosis entre lo que sería un plan director local con regulaciones específicas para el uso y la ocupación del territorio. Se constituye como el principal instrumento de orientación de la política de desarrollo urbano y de gestión territorial del paisaje cultural de Paranapiacaba. Adapta directrices de la conservación cultural y ambiental al desarrollo turístico, con el objetivo de lograr la sostenibilidad del patrimonio construido, natural e inmaterial de la villa, garantizando también la continuidad y la calidad de vida de los residentes.

Conforme a lo exigido en el Estatuto de la Ciudad (Ley Federal 10.257/01), el proceso de redacción del proyecto de ley se llevó a cabo de forma participativa, a través de la Comisión de la ZEIPP. Reuniendo a 34 miembros, con un $50 \%$ de representación de la comunidad local y las otras plazas para los representantes de los tres órganos de preservación patrimonial (nacional, estatal y municipal), el Consejo Municipal de Política Urbana, las universidades y las asociaciones profesionales, la comisión aseguraba la participación
3

Breve reseña sobre la ZEIPP publicada en la web del Ministério das Cidades/Secretaria Nacional de Programas Urbanos/Legislação. A invitación del IPHAN-Instituto do Patrimônio Histórico e Artístico Nacional, la ley se presentó en el I Fórum Nacional de Patrimônio (2009), en la mesa "Regulação e Marcos Legais". 
cualificada y activa de los representantes durante el proceso. Los técnicos de la administración municipal no tenían voto y participaban exponiendo los estudios técnicos y moderando los debates. Los técnicos de los órganos de preservación patrimonial participaban activamente desempeñando el papel de orientadores y cuestionando las propuestas.

Con respecto a la articulación específica entre las políticas de conservación patrimonial y las de planificación territorial, esta ley representa también un avance tanto en el marco teórico y conceptual como en la gestión. La villa de Paranapiacaba recibió, a través de una sola ley, un conjunto de directrices, instrumentos y parámetros urbanísticos y ambientales específicos para su realidad y articulados entre sí, lo que contribuyó a la institucionalización de una política adecuada a las singularidades de este territorio, en particular la conservación de la Mata Atlántica, la producción de agua, la preservación de una aldea de trabajadores con casas de madera y la vulnerabilidad social de la población residente.

Inicialmente, la ley establecía algunos conceptos relativos a la preservación, conservación, restauración, rehabilitación, mantenimiento, actualizaciones tecnológicas (retrofit) y adaptación (artículo 5), diferenciándolos y proponiendo una jerarquía de diferentes tipos de intervención en el patrimonio construido, destinada a reducir la burocracia en los procesos de aprobación, y el intercambio de roles entre las instituciones gestoras, institucionalizando una práctica fluida entre los distintos órganos competentes.

La ZEIPP ratifica la división de la villa en cuatro sectores de planeamiento urbano (Parte Alta, Parte Baja, ferrocarril y Rabique), reconociendo sus especificidades históricas, urbanas, paisajísticas y jurídicas. Crea una zonificación que prioriza el uso residencial y define áreas para el desarrollo de las actividades comerciales y turísticas, minimizando los conflictos vecinales. Funciona con control de inventario para la regulación de usos preferentes, como el APR-Área Predominantemente Residencial (zona predominantemente residencial), donde la concentración de usos relacionados com el comercio y los servicios de baja perturbación (hoteles y restaurantes abiertos hasta las 22h ) está permitida hasta un 20\%. En el APC-Área Predominantemente Comercial se permitien usos no residenciales hasta un $60 \%$. También establece en un $50 \%$ el conjunto de inmuebles públicos en la Parte Baixa, es decir, garantiza el derecho a la vivienda, evitando posibles modificaciones futuras que destinen los inmuebles a usos turísticos y casas de vacaciones, preferentemente. Igualmente fueron redefinidos los parámetros de ocupación de la parcelas (recuos) y sus límites, las tasas de permeabilidad, niveles de incomodidad para la emisión de ruidos, directrices para la conservación de los edificios y los espacios abiertos y la jerarquía del sistema de carreteras, con el fin de salvaguardar el conjunto y las relaciones urbanas que caracterizan el paisaje de la villa. 
Paranapiacaba sólo tiene 334 viviendas en la Parte Baixa. Fueron seleccionadas por ley propiedades representativas, designándolas como "Modelos de tipo de residencias". El objetivo era poner de relieve el valor documental y cognitivo del proyecto o la construcción original, sin que fuesen modificados, permitiendo que en las otras propiedades se llevaran a cabo intervenciones para la adaptación de usos conforme a las restricciones impuestas para cada tipo de inmueble.

Estos inmuebles-modelo estaban destinados a la visita pública, construyendo un circuito museológico, que fue premiado en 2007 por el IPHAN en el "concurso de modernización de museos". Basado en el concepto de "museo al aire libre", el proyecto articula espacios expositivos diversos en un recorrido que dibuja la noción de paisaje cultural: la casa "tipo C", conocida como "pequeño castillo" o "casa del ingeniero jefe" acoge una exposición sobre la historia de la villa; el patrimonio natural está expuesto en esta reproducción exacta de la casa del ingeniero. El patrimonio humano se encuentra en la "casa de la memoria", una copia de la casa "tipo A" (para las pequeñas familias de los operarios); un conjunto de casas "tipo E" (para trabajadores menos cualificados) se dedican al patrimonio arquitectónico y urbanístico; las casas "tipo D" integran ${ }^{4}$ el Antiguo Lyra da Serra (donde funcionó el segundo cine de Brasil), actualmente en restauración para albergar la sala de cine-teatro y un espacio para la educación patrimonial.

Para el resto de inmuebles están permitidas las modificaciones funcionales, siempre que respeten la relación entre espacios libres y edificados, según la norma urbanística de la villa, preservando la relación entre las parcelas, el cuerpo principal del edificio, el patio, el baño al fondo de la vivienda y los callejones auxiliares en medio de la cuadra. Es decir, para los inmuebles que originalmente tenían los baños fuera de la casa se permitiría la ampliación
4

Estas nomenclaturas fueron utilizadas por los ingleses en el caso de los planos y proyecto originales de la villa (LIMA, 2008).

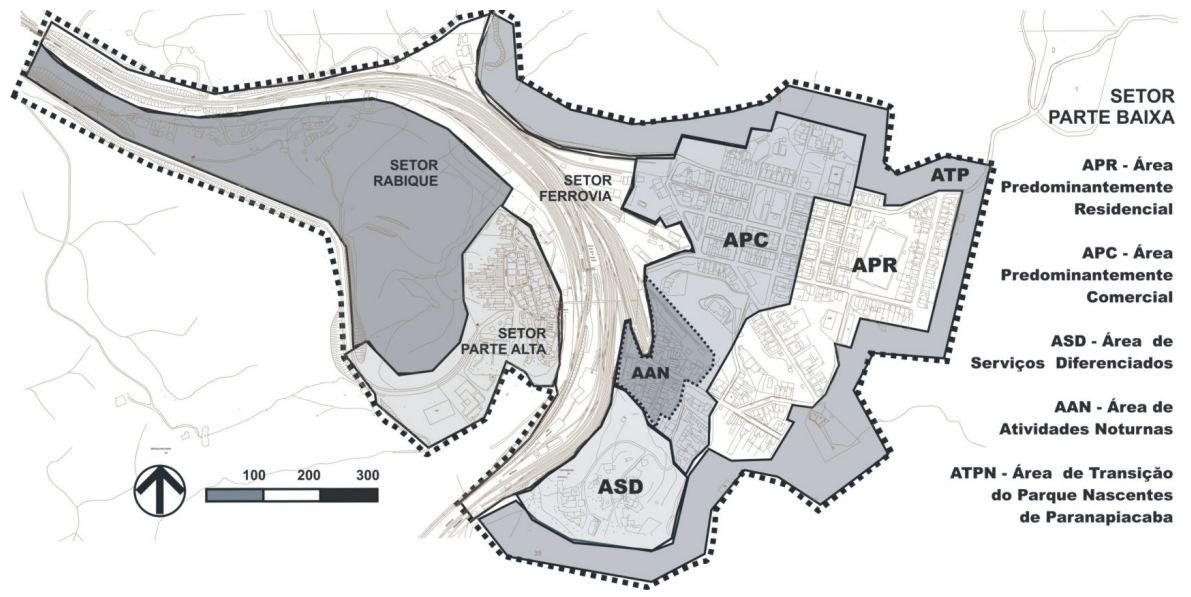

Paranapiacaba: zonificación | mapa Vanessa Figueiredo. Fuente Ley 9.018/07ZEIPP 
para cubrir necesidades tales como lavaderos cubiertos, siempre que no se incorporaran al cuerpo principal de la propiedad, a continuación del baño situado al fondo y siempre que no obstaculizaran los laterales y el frontal del edificio. De esta forma, se superó la aceptación de los niveles tradicionales de protección, entendidos como una gradación jerárquica y genérica incoherente con la concepción de paisaje cultural.

Se crearon instrumentos para incentivar la conservación y un sistema de supervisión y sanciones más riguroso, aunque adecuado a la realidad local. Con el objetivo de fomentar la conservación de los inmuebles y tener control sobre las acciones de los usuarios, se otorgaron descuentos como contraprestación para aquellos constructores con licencia que invirtiesen en rehabiltación, siempre y cuando se hiciera con el permiso del ayuntamiento y de los órganos de conservación. Este proceso de aprobación interinstitucional, que llevaba funcionando desde 2005, fue institucionalizado por ley.

La ZEIPP aseguró a los empresarios un instrumento de propiedad menos precario creando, para los inmuebles comerciales, la concesión de uso por 20 años, prorrogables por el mismo período. Asimismo, se estableció una nueva herramienta para el seguimiento y la democratización de la gestión: el Foro de Paranapiacaba, que reunía, de la misma forma que la Comisión ZEIPP, a representantes de la administración local, de las agencias de conservación y de la comunidad, en un debate permanente sobre el desarrollo sostenible de la villa.

La ley es, en su mayor parte, autoaplicable. Sólo algunos artículos necesitan una ordenación posterior, como el Foro (regulado en el 2008), o instrucciones normativas y planos, detallando información técnica que no se contempla en las leyes urbanísticas, como por ejemplo los manuales de arquitectura, paisajismo, y los planos de saneamiento, energía y alumbrado público, todos incluidos en 2008.

Paralelamente la administración municipal (subprefeitura) promovió diversas acciones para la conservación patrimonial, articulando investigación científica, sistemas de información y documentación, educación y formación profesional.

Esquema de las relaciones urbanísticas entre espacios libres y edificados del paisaje cultural de Paranapiacaba | gráfico Vanessa Figueiredo

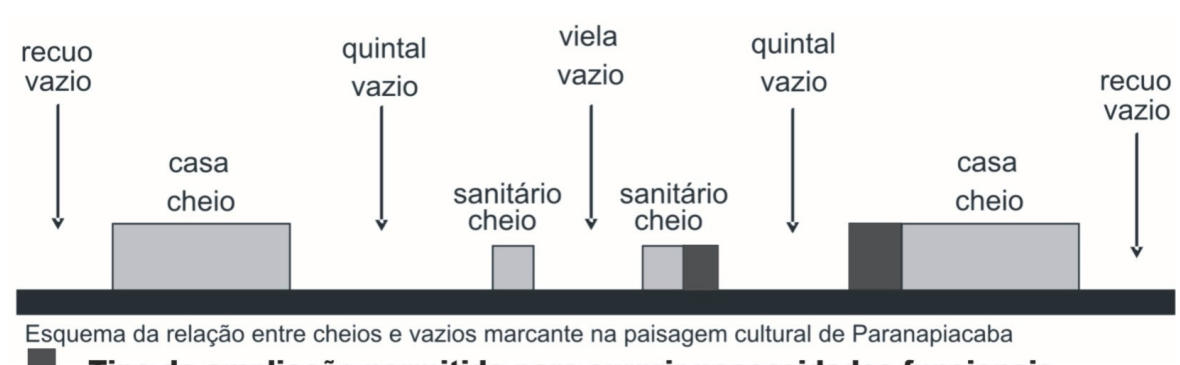

Tipo de ampliação permitida para surprir necessidades funcionais 
Entre 2004 y 2008, los investigadores del Centro Universitario de la Fundación San Andrés, financiado por la FAPESP-Fundación de Apoyo a la Investigación del Estado de São Paulo, área de políticas públicas y con el apoyo del ayuntamiento, desarrollaron la investigación "Directrices y Procedimientos para la Recuperación del Patrimonio edificado en madera de la villa de Paranapiacaba" (Lima, 2008). Este trabajo articula las necesidades de las políticas públicas municipales y obtuvo varias frutos, como la creación de una metodología propia para la catalogación del patrimonio en madera, la reactivación de la cooperativa de restauradores, la creación de un banco de materiales y la elaboración del Manual de Conservación y Restauración.

Todos estos resultados se incorporaron a la ZEIPP como directrices permanentes. El objetivo era proporcionar documentos-tipo que orientasen al personal técnico hacia procedimientos adecuados y respetuosos para las intervenciones en el patrimonio en madera. Cabe destacar que este es uno de los problemas más graves con los que se enfrentan los órganos de conservación en Brasil. La ausencia de directrices y parámetros preestablecidos capaces de orientar las intervenciones en edificios catalogados conducen a decisiones individualizadas, demasiado discrecionales y, muchas veces, antagónicas entre los diferentes organismos.

El inventario arquitectónico de inmuebles de la Parte Baixa fue sistematizado en la base digital "Banco de Datos de Gestión del Patrimonio Paranapiacaba", cruzando la información arquitectónica con los datos socio-económicos y administrativos de los residentes. Este inventario contiene información fotográfica, datos sobre la conservación de los inmuebles, así como la planimetría, su identificación según las tipologías, los anexos existentes y las paredes con materiales originales ya retirados o modificados de cada edificio.

La cooperativa de restauradores se formó con los residentes capacitados para trabajar específicamente en la restauración y conservación de madera. En el año 2008, ya se había restaurado un conjunto de cuatro casas "tipo E", una casa de ingeniero que había ardido fue restaurada gracias al programa de la biblioteca pública, el recinto de un bloque y la antigua panadería, terminadas en 2010. Además, la cooperativa producía elementos constructivos, tales como puertas, ventanas, soportes tipo "manos francesas", aleros y vallas, destinados al establecimiento de un banco centralizado de materiales para la reposición adecuada de los elementos arquitectónicos degradados.

A partir de 2006 se promovieron cursos específicos de educación patrimonial $^{5}$, cuyo módulo básico era ofrecido a todos los residentes y el resto de módulos (intermedio y avanzado) se destinaban a formar a monitores culturales.
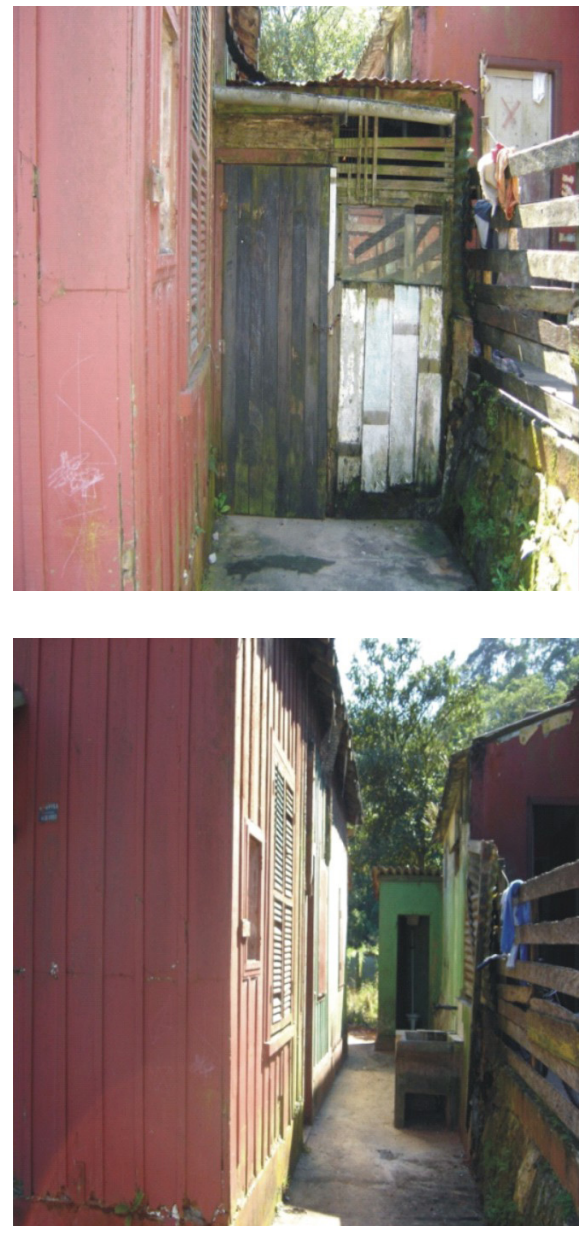

Eliminación de los anexos irregulares o en malas condiciones | fuente Prefeitura Municipal de Santo André, 2007

5

Curso impartido en colaboración con los órganos de defensa del patrimonio (IPHAN, CONDEPHAAT Y COMDEPHAPASA), el Museu de Santo André, el MAE-Museu de Arqueologia e Etnologia da USP, la ABPF-Associação Brasileira de Preservação Ferroviária y la Fundação Santo André/FAPESP. 
6

Con recursos del Ayuntamiento, de la American Express a través de la World Monuments Fund, de la Petrobrás, de la FAPESP/Centro Universitário Fundação Santo André y de Ministério do Turismo.
Tratando de revertir el proceso de degradación sufrido en los años 80 y 90 , del siglo XX, la administración local retiró, hasta 2008, un total de 49 anexos precarios e irregulares de los inmuebles, el 50\% de la demanda. El procedimiento tenía lugar cuando las propiedades eran devueltas o cuando se llegaba a un acuerdo con los lugareños. La ley de ZEIPP estableció que una acción más ambiciosa fuese iniciada en 2010. Sin embargo, el nuevo gobierno podría ampliar este plazo y reanudarlo de forma efectiva con las obras del PAC-CH a partir de 2015, ya que estaban previstas las intervenciones de 242 casas.

Finalmente, entre 2001 y 2008, se invirtieron cerca de 4,5 millones de dólares en 27 obras de restauración del patrimonio edificado y espacios libres ${ }^{6}$, además de la inversión anual de 82.000 dólares en conservación y mantenimiento continuo.

\section{EL TURISMO SOSTENIBLE Y LA CONSERVACIÓN MEDIOAMBIENTAL}

El programa propuesto preveía la promoción del turismo de base endógena, donde la comunidad estuviese inmersa en la rutina de la visita pública, la convivencia con el viajero y las actividades y productos turísticos. Este proyecto se llevó a cabo con planificación y de forma paulatina, con el fin de incluir a los residentes y evitar posibles impactos no deseados sobre el patrimonio, el medio ambiente y la rutina y la calidad de vida de la población.

El ayuntamiento utilizaba un método de planificación estratégica situacional que fue estructurando el programa en tres etapas. El objetivo de la primera etapa (2001-2004) fue la implantación de la actividad turística, con la creación de la infraestructura de recepción, manutención, alojamiento, y resto de productos y servicios turísticos, prácticamente inexistentes. Varias actividades promovían la inclusión de la comunidad en la actividad turística, tales como: puertas abiertas, Fog \& Fogão, Bed and Breakfast y el AtelierResidencia. Todo ello alentando el uso mixto del inmueble, es decir, el residente podía abrir un proyecto orientado al turismo en su propia casa. Como incentivo financiero, aquellos que se unían al programa disfrutaban de un descuento del $70 \%$ en los alquileres. El principal resultado fue un salto de 9 proyectos en 2002 a 97 en 2008. Asimismo, se instituyó el cálculo de visitantes que registró en 2002 unos 41.000 turistas anuales, y 220.000 en 2008.

En 2003 se elaboró el "Plan Patrimonio", la sistematización de un diagnóstico de los atractivos turísticos, creando una marca, un logo y unas estrategias. En ese momento se concibieron dos productos principales para Paranapiacaba: la Agenda cultural anual y el Parque Nascentes. 


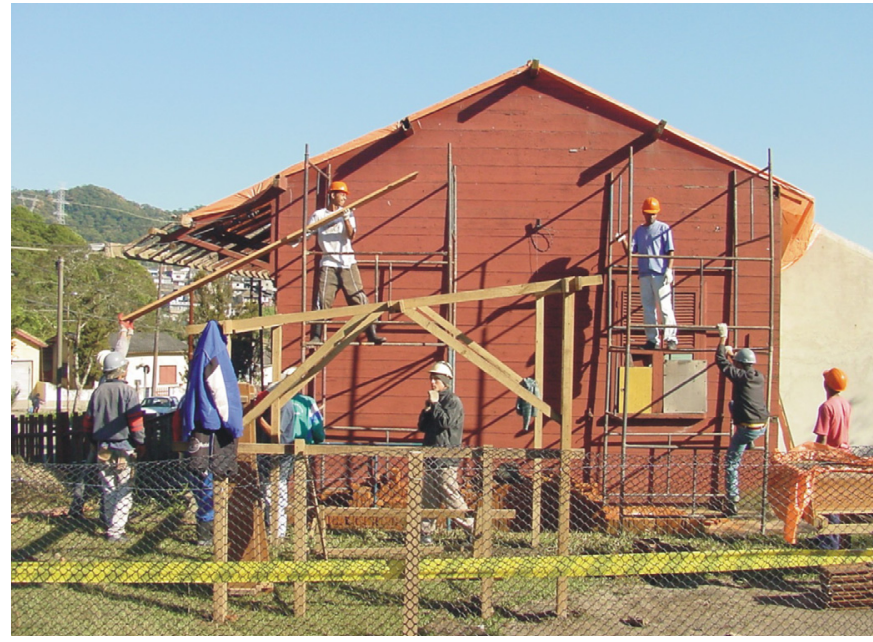

La Agenda consistía en una combinación que buscaba promover tres tipos de eventos: aquellos tradicionales que ya practicaban la comunidad y la región; los eventos nacionales naturales; y aquéllos diseñados para atraer al público. De ese modo, el año comienza con la fiesta brasileña más tradicional: el carnaval. En abril se celebra el Festival Gastronómico de Cambuci (productos típicos de la Mata Atlántica identificados en la práctica culinaria a través de estudios sobre la historia oral), seguido por el Festival de junio y las celebraciones mes del medio ambiente. En julio tiene lugar el evento principal del calendario, el Festival de Invierno, que representa alrededor del $50 \%$ de la visitas anuales. En agosto es la fiesta del patrón, le sigue la Semana del Ferrocarril, el mes de la infancia y de la Feria de los altares y belenes. En 2004 se celebró el primer Festival de brujas y magos, una importante comunidad organizada a nivel nacional que tiene en Paranapiacaba, debido a sus propiedades místicas, el lugar de sus costumbres y tradiciones. Esta práctica está en la línea de lo que el Comité del Patrimonio Mundial ha estado llamando paisaje cultural asociativo. También en 2008 se desarrolló el primer Festival de Cine de Paranapiacaba, y ambos se incorporaron al calendario anual de eventos.

El Parque Municipal Natural Nascentes de Paranapiacaba, surgido en 2003, es una Unidad de Conservación con 4,2 km², que ofrece senderos, arboleda e interpretación ambiental del bosque. Limita con dos áreas protegidas (UCs): la Reserva Biológica do Alto da Serra de Paranapiacaba y el Parque Estadual da Serra do Mar. El área se convirtió, en 2008, en una de las zonas más conservadas de la Reserva de la Biosfera del Cinturón Verde de São Paulo, formando un amplio corredor ecológico que comprende 73 municipios, y cuyo objetivo común es la conservación y la gestión integrada de la biodiversidad y los ecosistemas del Atlántico.
Cooperativa de carpintería/ebanistería. Oficina del banco de materiales y restauración de las casas tipo E-CDARQ | fotos Gilson Lameira de Lima, 2007 (LIMA, 2008) 


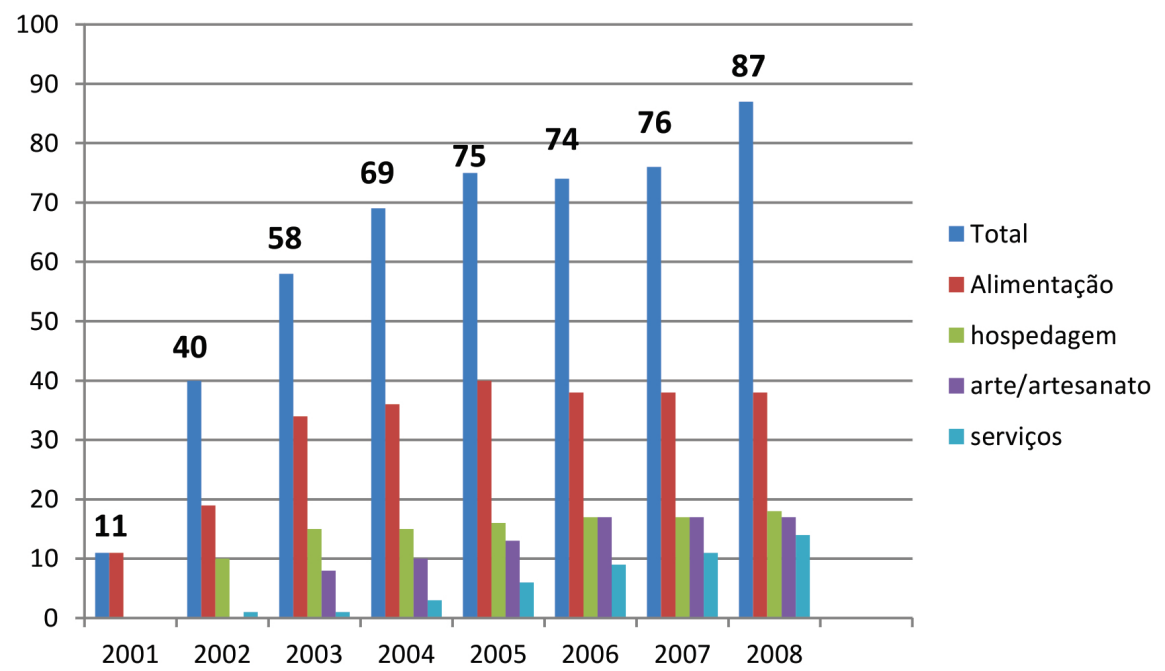

Negocios abiertos en la Villa de Paranapiacaba, 2001-2008 | fuente FIGUEIREDO; RODRIGUES, 2014

El parque contribuye de manera importante a la formación de reservas de agua que alimentan el embalse Billings. Hasta el 2008 pasaron por él 90.000 visitantes, acompañados de monitores ambientales que habían sido formados por el programa para el turismo, promovido por el ayuntamiento en colaboración con el Instituto Forestal. A medida que el parque se fue cerrando, para garantizar el acceso de los residentes, la administración local creó el programa "Amigos del Parque", con la emisión de 300 tarjetas de identificación. A los grupos de ingresos reducidos y a las escuelas públicas se les ofrecieron 600 visitas gratuitas mensuales. También se celebraron eventos de turismo deportivo y de aventura, tales como carreras de montaña y trekking. En 2004 se publicó la primera edición del Atlas del parque, con estudios sobre los aspectos naturales. En 2008 se publicó la segunda edición ampliada que también incluía propuestas para el plan de gestión.

La segunda etapa del programa de turismo sostenible (2005-2008) se ocupó de la calificación de calidad de los proyectos abiertos, con la organización de actividades urbanas en el territorio (ZEIPP) y la integración final de las políticas sectoriales. En este contexto surgió el PQST-Programa de Calificación de Servicios Turísticos, que ofrecía distintos cursos de educación ambiental, patrimonial, de formación profesional, en lenguas extranjeras, gastronomía, cooperación y espíritu empresarial. Estos cursos formaron, hasta 2008, a 50 monitores ambientales y 30 culturales. La participación en el PQST era opcional, pero proporcionaba un sistema de evaluación a través de la Certificación 5. Los niveles que calificaban las empresas oscilaban desde uno a cinco vagones ferroviarios, sustituyendo a la tradicional gradación por estrellas. En 2005 se hizo la primera certificación y en 2008 la segunda, lo que permitió la evaluación periódica de la calidad de los servicios turísticos y de la participación comunitaria en los cursos y proyectos ofrecidos, 
fomentando la responsabilidad de cada uno en la construcción del proyecto colectivo.

El Plan de Patrimonio se revisó en 2007 sobre la base de las directrices de la ZEIPP, generando el PDTUR-Plan de Desarrollo turístico sostenible. El PDTUR reevalúa atractivos y productos turísticos, así como los segmentos a los que se les debe dar prioridad. Paranapiacaba pasa entonces a centrarse en cuatro segmentos: turismo cultural, ecoturismo, turismo educativo y turismo de calidad de vida. También se trabajan, como segmentos secundarios, el turismo deportivo, de aventura y demás eventos. EI PDTUR estableció un plan de infraestructura turística, un plan de comunicación y un plan operativo.

A partir de 2007, con la aprobación de la ZEIPP, comenzó la preparación de la tercera fase del proyecto, que incluiría la formalización y la regulación de empresas turísticas. Este proceso visaba la adecuación de las empresas a los requisitos legales, como el control sanitario, las licencias de obra, además de la legalización de los trabajadores. Sin embargo, este paso no se completó debido al cambio de la administración municipal en 2009.

Durante todo este tiempo, se buscaron alianzas para hacer viable el retorno del tren a Paranapiacaba, inactivo desde 2002. En el año 2006 fue posible la implantación de una locomotora de carbón en el patio de ferrocarril y en 2008 se firmó un acuerdo de cooperación entre los gobiernos de San Andrés y Jundiaí, la Secretaría de Transporte del gobierno del Estado de São Paulo, la compañía paulista de trenes metropolitanos, el IPHAN y la Asociación Brasileña de preservación ferroviaria para el tren turístico expreso. En el mismo año se realizó el viaje inaugural entre las estaciones de Luz (São Paulo)-Jundiaí, y de Luz-Mogi das Cruzes. En 2010 se inició la operación Luz-Paranapiacaba. Todas las partes tenían como guías turísticos a los monitores formados en la villa.

\section{EL DESARROLLO SOCIAL Y LA PARTICIPACIÓN CIUDADANA}

Entre 2001 y 2008, se implantó un modelo de gestión democrática y participativa que colocaba a los ciudadanos en la primera línea de la toma de decisiones locales. Un modelo que no fue construido de forma centralizada, sino que se sustentaba en redes de participación ciudadana organizadas con los distintos actores locales y cuyo centro de decisiones estaba siempre en la propia villa, apoyados por los poderes públicos, las instituciones afines, los organismos encargados de las podíticas urbanas y ambientales de conservación, los ciudadanos y demás interesados.

Sin embargo, la implementación de este nuevo modelo encontró barreras que exigían a los directores una revisión constante del proceso y las habi- 
lidades para poderlas superar. Estas dificultades pasaban por la falta de confianza de la población residente en el gobierno, ausente durante mucho tiempo, y por la interrupción de los privilegios establecidos en los últimos años por dirigentes que sacaban provecho del estado de desorden físico, social e institucional con el que se encontraban (MORETTO, 2005).

Por lo tanto, la implementación de estas políticas se llevó a a cabo con la comunidad, a menudo con demasiados debates y conflictos, inherentes al proceso democrático y al establecimiento de un nuevo orden socio-institucional, con la participación de un gobierno comprometido con el desarrollo local sostenible.

Se crearon tanto instancias participativas diversas, como el Presupuesto Participativo y los Consejos temáticos, hasta otras específicas, como el Consejo de Representantes. Con la compra de la villa se creó un fondo público para su gestión y para recibir el dinero de los alquileres de los arrendatarios de los inmuebles. EI FUNGEPHAPA-Fondo de Gestión del Patrimonio Histórico de Paranapiacaba- recibe recursos provenientes de la utilización de los espacios institucionales, del uso comercial de la imagen de la villa y de las instituciones financieras. Cada dos años se realizaba la renovación del contrato y las irregularidades eran remitidas a la administración sancionadora correspondiente, estableciéndose tres pasos para llegar a un acuerdo sobre la deuda o, en última instancia, la restitución de la propiedad por orden judicial. En el año 2008 fueron ejecutadas 24 devoluciones, sobre todo de aquellos que se negaron a reconocer la propiedad pública y de los que estaban involucrados en actividades ilícitas, como tráfico de drogas, prostitución infantil y robo. Todos lo que tenían que abandonar sus hogares recibían un alquiler social durante seis meses.

En 2008, el FUNGEPHAPA, que era gestionado de forma consensuada con la comunidad por medio del consejo, aumentó sus ingresos en un $76 \%$ respecto a 2002, con una recaudación anual de 340.000 dólares (PMSA/SUB, 2008). Estos recursos fueron utilizados para la conservación de edificios, de la reserva natural, para los cursos y para la promoción del turismo.

Para responder mejor a los deseos de la comunidad, el gobierno sintió durante el proceso la necesidad de crear instancias más específicas de participación, centrándose en temas de mayor interés y más demandados, tales como: encuentros de monitores, de empresarios; las Comisiones de festejos y de la ZEIPP. Estos órganos de gestión participativa estaban encaminados a integrar los distintos saberes y buscar soluciones alternativas conjuntas a los problemas cotidianos, programas y planes, involucrando a la comunidad en la corresponsabilidad en las decisiones y actuaciones políticas.

Por otro lado, estos sistemas fomentaban la participación permanente y el protagonismo de la comunidad, impulsando una estructura de gobernanza 
local, cuyo objetivo era también garantizar la continuidad de la conservación más allá de la acción directa de la administración pública, es decir, partiendo del proprio compromiso social.

Además de estos mecanismos de participación, se alentó a la comunidad a invertir en su cualificación y generación de ingresos. Dentro del PQST, se promovieron cursos de formación profesional fomentando la organización de grupos a través de cooperativas o asociaciones. Se impartieron cursos de economía solidaria, carpintería, ebanistería, restauración de madera, artesanía en cerámica, grabado en madera, gastronomía, electricidad, albañilería y fontanería. En 2008 estaban en funcionamiento la Cooperativa de restauración en madera y tres asociaciones de servicios turísticos: la AMA (Asociación de Monitores Ambientales Paranapiacaba); la ECOVERDE y ECOPASSEIOS. Participaban de la formación la cooperativa de gastronomía y la asociación de artesanos. Otros cursos buscaban la formación continua y el perfeccionamiento de los monitores de las acciones culturales, como el de "Aprendizaje secuencial y experiencia en la naturaleza" o el de la "Memoria Oral" para la formación de "Agentes de la Memoria", cuya primera actividad consistió en una exposición presentada en la Casa Fox.

Se invirtió especialmente en la formación y la inclusión de los jóvenes a través del Programa Juventud de la Reserva de la Biosfera (PJ) y de Agente de la Juventud, desarrollado en colaboración con el Instituto Forestal y la UNESCO. PJ buscaba, además de la formación integral de los adolescentes entre 14 y 21 años, la capacitación para la gestión ambiental, el ecoturismo, la silvicultura, la agroindustria artesanal, el arte y el reciclaje.

Hasta el año 2008 el programa atendió a cerca de 220 jóvenes trabajando la autoestima y la formación del carácter, además de contribuir a su inserción en el mercado laboral. Ya en esa fecha (2008), 14 jóvenes habían sido contratados como monitores del Parque Estadual Caminhos do Mar, otros 20 desempeñado tareas como monitores ambientales en Paranapiacaba y el $100 \%$ tuvieron oportunidades de trabajo durante los eventos turísticos anuales. Cinco continuaron estudios en las facultades de biología, gestión ambiental, administración y educación física. En 2006 se pusieron en marcha el vivero y el jardín experimental, financiados por el Banco Mundial (PMSA/SUB, 2008).

Paralelamente, el programa Agente Joven, en colaboración con el Gobierno Federal, proporcionaba formación socioambiental a jóvenes entre 14 y 17 años de toda la región de los manantiales. De la villa se formaron 40 alumnos, 13 de ellos recibieron becas por valor de 30 dólares mensuales.

Para promover la inclusión de aquellos residentes que no tenían posibilidades socioeconómicas para abrir empresas sin ayuda, se creó el "Almacén de

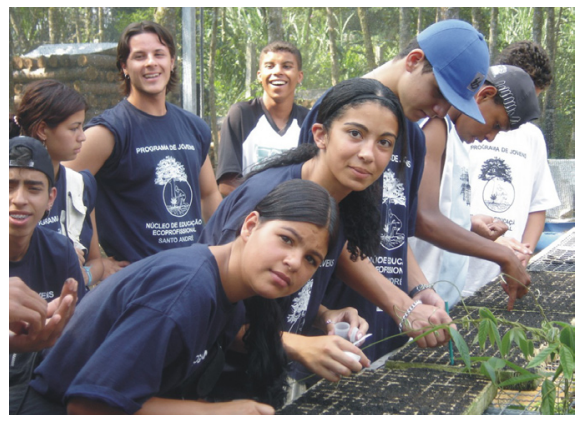

Programa parajóvenes (UNESCO, Gobierno delEstado y Ayuntamiento) | fuente PMSA/Subprefeitura, 2005 
arte y artesanía" y el "Espacio Gastronómico". Al principio, el espacio cedido como sede de ambos proyectos fue el viejo mercado y en 2006 recibieron dos inmuebles para sus actividades. A los empresarios residentes en la villa se les concedió el $70 \%$ de descuento en los alquileres, ya que este proyecto incluía también a los residentes de la Parte Alta y de la región.

En caso de tener constancia de situaciones de riesgo social, es decir, familias numerosas en casas pequeñas, varias familias compartiendo el mismo inmueble o alquileres incompatibles con la renta familiar, e incluso riesgo ambiental (geotécnico, inestabilidad estructural o condiciones insalubres), el ayuntamiento procedía a la reubicación de estas familias en otras propiedades que podrían proporcionar una situación socioambiental más adecuada.

Además, la población con ingresos bajos estaba incluída en los programas de transferencia de ingresos. La Bolsa Familia, la Familia Andreense y la renta Ciudadana atendían a 32 familias de la villa y 320 en la región.

Para evaluar y monitorizar las políticas sociales en 2005 se realizó un catastro socioeconómico y cultural completo de los residentes y encuestas puntuales entre 2007 y 2008. Algunos indicadores confirmaron el impulso del desarrollo local (LUME, 2000; MORETTO, 2005; PMSA/SUB, 2008):

$>$ La renta media individual aumentó en $77,58 \%$, de 85 dólares en 2001 a 150 en 2005; los monitores ambientales y culturales ganaban un promedio de 340 dólares mensuales en temporada alta; el desempleo se redujo del $61 \%$ en 1999 al $30 \%$ en 2005 y $16 \%$ en 2008; el número de empresarios aumentó de apenas 9 en 2001 a 97 en 2008 y el 90\% de ellos eran residentes de la villa; el volumen de negocios anual medio declarado por los empresarios subió más de $3.000 \%$, desde 18.000 dólares/año en 2002 a 565.000 en 2007.

> También hubo un aumento en los niveles de escolarización; el número de escolarizados en $2^{\circ}$ grado subió de 20,21 \% en 1999 a 56,65\% en 2005 y en el superior del $2 \%$ al $5 \%$ en el mismo período. Hay que destacar que muchos de los jóvenes que cursaron $3^{\text {er }}$ grado eligieron opciones relacionadas con las actividades ambientales y el turismo.

\section{LEGADOS, ENSEÑANZAS, PERMANENCIAS Y RUPTURAS}

Bajo este amplio programa de desarrollo local sostenible, basado en la integración entre las políticas públicas y el debate permanente entre la comunidad y las instituciones, la experiencia de Paranapiacaba se enfrentó con supuestos y desafíos establecidos en reflexiones académicas y, por sus resul- 
tados y logros, se destaca como un modelo de gestión no solamente necesario para las ciudades, sino posible para la administración pública local.

Por eso, ha sido considerado innovador por varias instituciones nacionales como el IPHAN, el Ministerio de las Ciudades y la Reserva de la Biosfera del Cinturón Verde de São Paulo, y por organismos internacionales como el Programa Mundial de Estudios del Patrimonio de la Universidad Tecnológica de Brandeburgo en Alemania (World Heritage Studies da Brandenburg University of Technology) y el Laboratorio Internacional de Paisajes Culturales de la Universidad Politécnica de Cataluña. Así, entre las principales conclusiones y enseñanzas aportadas por esta experiencia hay que destacar diez:

$>$ En primer lugar, de vital importancia para la puesta en marcha y el funcionamiento del programa, está la existencia de una estructura de gestión descentralizada, con autonomía administrativa y presupuestaria. Esto implica el reconocimiento de las especificidades del territorio, el diálogo de proximidad con su población, la integración de políticas gestionadas dentro de la misma estructura organizativa y un cuerpo técnico interdisciplinar, así como la independencia de aquellos sectores de la administración central que no tienen a la región como una prioridad.

$>$ En segundo lugar, la implementación de un sistema permanente de planificación y evaluación. En este caso, se utilizó el método de la planificación estratégica situacional, instrumento que posibilitó la revisión constante de las políticas, la detección y resolución de los problemas, el reajuste de los objetivos, la incorporación de oportunidades, la integración de acciones en las distintas áreas y la mejora de los resultados.

$>$ El tercer punto a destacar es la importancia de la integración de las políticas sectoriales con el objetivo de alcanzar la interdisciplinariedad. La colaboración del personal técnico de la administración local fue fundamental para el éxito de los programas, proyectos y acciones.

> La cuarta clave radica en un sistema de participación cualificada, que por un lado capacita a la comunidad para el debate y, por el otro, fomenta la articulación de una red de colaboradores y líderes comprometidos con las políticas puestas en práctica.

$>$ El quinto punto se refiere al uso de los recursos naturales y culturales de una manera sostenible, a favor del desarrollo socio-económico local, contrastado en los indicadores cualitativos y cuantitativos dados a conocer.

$>$ El sexto se refiere a la política de inclusión social estratégicamente desarrollada por etapas, acorde en todo momento a las necesidades y condiciones 
socioeconómicas de la comunidad, pero requiriendo su corresponsabilidad. Es decir, contemplando un primer momento de tutela, incentivo y adhesión al programa, o, literalmente, "proporcionando los peces"; y una segunda etapa que exige la cualificación de los participantes y su responsabilización en el proceso de construcción colectiva del proyecto "enseñando a pescar", para terminar con la emancipación a través de la formalización (fase todavía incompleta).

$>$ El séptimo punto hace hincapié en la importancia del trío: planificación, formación y fiscalización. A pesar de toda la inversión en educación (ambiental, cultural, formación profesional, progreso escolar) y planificación (territorial, participativa y de las políticas sectoriales), éstas no se sustentan sin la fiscalización, sin supervisión, que juega un papel también pedagógico en la medida en que disciplina la acción humana a favor de los intereses comunes y generales.

$>$ El octavo muestra que la preservación cultural requiere mucho más que acciones encaminadas a la restauración, especialmente en el caso del enfoque territorial, y más si se incorpora una aproximación al paisaje cultural. Las dimensiones social, urbana, ambiental, económica y política no pueden ser ignoradas por los órganos responsables. Sin embargo, como no hay en Brasil, todavía, un único órgano que tenga competencia para abordar a todas estas áreas, hay que buscar la colaboración interinstitucional.

$>$ El noveno punto se refiere precisamente a la importancia de esta articulación interinstitucional. En el caso de la gestión ambiental, se desarrollaron varios proyectos conjuntamente con el Gobierno Central, el Instituto Forestal, el Comitê de Bacias y la Reserva de la Biosfera (UNESCO), cumpliendo las directrices nacionales (resoluciones de CONAMA). En el caso de la preservación cultural, aunque ésta sea una experiencia puntual, la corresponsabilidad impulsada por un sistema de aprobación conjunta de proyecto, así como por los procesos de elaboración y aplicación de la ZEIPP, permitieron la articulación conjunta de las directrices de conservación entre los tres órganos implicados. Sin embargo, es esencial reconocer que la consolidación de este nuevo acuerdo institucional no vendrá dada por las decisiones y actuaciones de los municipios por muy beneficiosas que sean. Estos desafíos deben ser asumidos y conducidos a través de la construcción de un marco jurídico que establezca un Sistema Nacional de Patrimonio, que permita la acción compartida y corresponsable en los tres niveles de gobierno.

> Por último, es importante tener en cuenta que a partir de 2009, debido a la ruptura que se produjo con el cambio de gobierno municipal, hubo una desarticulación de la gestión local en marcha, debido principalmente a la ausencia de un plan de gobierno, la inexperiencia del nuevo equipo gestor, la abolición de la subprefeitura, y del sistema de participación. Sin embargo, 
aunque para el visitante sea evidente el desmantelamiento del programa, es posible constatar la supervivencia de algunos proyectos e instrumentos que fueron capaces de consolidar parte de las políticas implementadas.

En primer lugar hay que destacar la permanencia de instrumentos jurídicos, como la ZEIPP, la Unidad de conservación y los contratos existentes para obras de restauración. La existencia del Parque Nascentes y su Plan de Gestión, cuya segunda etapa continuó desarrollándose, y se terminó en 2011, propiciaron la conservación ambiental y el ecoturismo sostenible. La ZEIPP, aunque con retrasos en su aplicación e interpretación erróneas aseguró la ordenación del turismo y el mantenimiento del uso residencial, así como los criterios generales para la conservación.

También hay que señalar la inversión en la formación de recursos humanos dentro de la propuesta de desarrollo endógeno, pues la actividad turística de cada día fue realizada efectivamente por los monitores culturales y ambientales, por los empresarios cualificados, y por las instituciones organizadas durante el gobierno anterior, ya que el cuerpo de funcionarios públicos habilitado para conducir el proyecto fue desmantelado. La Cooperativa de Restauración que sobrevivió, en un primer momento debido a los contratos en curso, fue desmontada por la nueva administración, con el objetivo de desmantelar las redes y organizaciones sociales promovidas anteriormente. Sin embargo, debido a la escasez de mano de obra cualificada para la restauración de la madera, los trabajadores de la cooperativas fueron subcontratados por la empresa que ganó la licitación de la restauración de Antigo Lyra da Serra en 2011. El trabajo fue paralizado en 2012 debido a problemas de gestión y se reanudará en este año 2015 a través del PACCH.

Otros supervivientes fueron los proyectos consolidados, enraizados en la rutina, expectativas y en la memoria de la comunidad y de los visitantes asiduos, que se habían convertido en tradiciones, como el Festival de Invierno, las rutas de ecoturismo y el Festival de Cambuci que, por la acción de la Incubadora de Proyectos Sociales de Ayuntamiento de São Paulo, llegó a ser regional en 2009, con la participación de ocho ciudades. El Circuito museológico continuó existiendo; sin embargo, la visita completa se realizaba mediante cita previa, ya que los monitores, sin apoyo institucional, no consiguieron mantener abiertos de manera permanente los espacios expositivos.

Por otro lado, a pesar de que hubiese sido fundamental la inversión y la vuelta a los procesos participativos de gestión, se constató que no era capaz de generar el empoderamiento permanente de la población ni de mantener la red de actores comprometidos que se había fomentado. Tanto las instituciones organizadas como los ciudadanos individuales se sintieron acorralados por el absolutismo del nuevo gobierno y por las amenazas constantes 
7

Como desvela el artículo "Vila de Parana piacaba é retrato do descaso" (trad: Villa de Paranapiacaba es la imagen del abandono). Santo André: Jornal Diário do Grande ABC. [En línea] <http://www.dgabc.com.br/ News/5847239/vila-de-paranapiacaba-e-retrato-do-descaso.aspx> [Consulta: 5/2/2012] También constatado por la autora en una visita a la villa. de desalojo, un proceso que llevó a la aparición de liderazgos nocivos que, democráticamente, también podían participar en el proceso.

Esta experiencia vuelve a colocar el acento sobre un aunto: la capacidad emprendedora y articuladora del poder local, por supuesto siempre dentro de sus competencias. Como consecuencia del proceso y de los resultados obtenidos por el programa y de los efectos nefastos que acarreó de manera inmediata el desmantelamiento de esta política, cabe una profunda reflexión sobre el papel del poder local. Tales efectos adversos pueden ser constatados por la disminución de las visitas turísticas, que se redujeron hasta en un $70 \%$, o por los traslados ${ }^{7}$, produciendo el cierre oficial de 18 tiendas y dejando muchos edificios vacíos entre 2009 y 2012. Por otra parte, este hecho pone de manifiesto la incapacidad de los óganos de conservación cultural para actuar ante tal situación. Mientras que la corporación municipal vio su acción limitada por la governanza local, los poderes federal y estatal se distanciaron de la gestión y del día a día de los ciudadanos, aunque no faltaron denuncias e incluso la injerencia del Ministerio Público en algunos casos graves relacionados con las propiedades legítimas de los ciudadanos.

No obstante, el último proceso electoral reveló la insatisfacción de los andreenses reemplazando a parte del grupo político anterior en el gobierno. Sin embargo éste estudia con calma la restitución de la subprefectura e intenta la reorganización de gestores, emprendedores y la comunidad que, conjuntamente, implantaron el programa en el terreno.

Del sistema político propiciador de la continuidad o el cambio hay poco que decir; debemos destacar simplemente que se trata de un proceso aparte, diferenciado de la gestión administrativa y que sufre los efectos inesperados de factores coyunturales. Además, forman parte del proceso democrático tanto el cambio como la continuidad, así como una agenda de prioridades elegida por cada programa designada para cada mandato. Parafraseando a Churchill, "la democracia es el peor sistema de gobierno, a excepción de todos los demás". Dejemos, pues, todo lo demás en el cajón y sigamos recorriendo el intrépido camino de las elecciones y sus consecuencias. 


\section{BIBLIOGRAFÍA}

- CONSEJO DE EUROPA (1995) Recommendation No. $R$ (95) 9 of the Committee of Ministers to Member States on the Integrated Conservation of Cultural Landscape Areas as Part of Landscape Policies, 1995 [en línea] <https://wcd. coe.int/wcd/> [Consulta: 11/10/2011]

- FIGUEIREDO, V. G. B. (2014) Da Tutela dos Monumentos à Gestão das Paisagens Culturais Complexas: inspirações à política de preservação cultural no Brasil. São Paulo: FAU USP, 2014 (Tesis doctoral)

- FIGUEIREDO, V. G. B.; RODRIGUES, R. (orgs.) (2014) Paranapiacaba: um patrimônio para a humanidade. São Paulo: Editora Marquise, 2014

- LIMA, G.; AZEVEDO, M.; PASSARELLI, S. (2008) Diretrizes e Procedimentos para a Recuperação do Patrimônio Habitacional em Madeira na Vila Histórica de Paranapiacaba. FAPESP/ PMSA/ Fundação Santo André. Santo Andre: [s.n.], 2008 (Informes de la investigación)

- LUME (Laboratório de Urbanismo da Metrópole) (2000) Plano de Desenvolvimento Sustentável da Vila de Paranapiacaba-SANTO ANDRÉ. Etapa 2-Produto Final. São Paulo: FAUUSP, 2000

- MORETTO, M. (2005) Protagonismo comunitário em Paranapiacaba: o impacto das ações governamentais no desenvolvimento sócio-econômico-comunitário da Vila de Paranapiacaba no período de 2001 a 2004. São Caetano do Sul: Universidade de São Caetano do Sul, 2005 (tesis de master)

- PMSA/SUB (Prefeitura Municipal de Santo Andrél Subprefeitura de Paranapiacaba y Pq. Andreense) (2006) Memorial da ZEIPP-Zona Especial de Interesse do Patrimônio de Paranapiacaba. Santo André: [s.n.], 2006

- PMSA/SUB (2008) Relatórios de Gestão do Departamento de Paranapiacaba 2001-2008. Santo André: [s.n.], 2008

- PMSA/CIDA (Prefeitura Municipal de Santo André/ Agência Canadense para o Desenvolvimento Internacional) (2004) Desenvolvimento Econômico Comunitário e Turismo para Inclusão Social-Projeto GEPAM. Vol 5. São Paulo: Annablume, 2004 